\title{
eJRIEPS
}

Ejournal de la recherche sur l'intervention en éducation physique et sport

Numéro spécial 2 | 2019

Actes du congrès ARIS 2016

\section{L'apprentissage paritaire en EPS : analyse comparatiste des interactions dyadiques en handball et danse}

Peer Learning in Physical Education: a comparative analysis of handball and dance dyadic verbal interactions

\section{Nada Ghorbel et Florence Darnis}

\section{OpenEdition}

Journals

Édition électronique

URL : http://journals.openedition.org/ejrieps/939

DOI : 10.4000/ejrieps.939

ISSN : 2105-0821

Éditeur

ELLIADD

Référence électronique

Nada Ghorbel et Florence Darnis, «L'apprentissage paritaire en EPS : analyse comparatiste des interactions dyadiques en handball et danse », eJRIEPS [En ligne], Numéro spécial 2 | 2019, mis en ligne le 01 juillet 2019, consulté le 01 août 2019. URL : http://journals.openedition.org/ejrieps/939 DOI : 10.4000/ejrieps.939

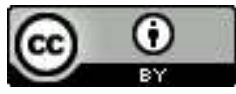

La revue eJRIEPS est mise à disposition selon les termes de la Creative Commons Attribution 4.0 International License. 


\title{
L'apprentissage paritaire en EPS : analyse comparatiste des interactions dyadiques en handball et danse
}

\author{
Nada Ghorbel* \& Florence Darnis ${ }^{* *}$ \\ * Laboratoire LACES, UFR STAPS Bordeaux, Université de Bordeaux, France. \\ ** Laboratoire LACES, ESPE Aquitaine, Université de Bordeaux, France.
}

\section{Résumé}

Cette étude repose sur une approche intégrative des travaux post-piagétiens (Muller-Mirza et Perret-Clermont, 2008) et de Vygotsky pour des recherches sur les apprentissages scolaires (Brossard et Fijalkow, 1998 ; Vergnaud, 2002). II s'agit d'envisager la construction de compétences spécifiques et transversales en EPS en étudiant le rôle du langage en tant qu'outil conceptuel. Nous situons notre travail dans une perspective comparatiste en étudiant les interactions verbales paritaires des élèves dans une classe de 6ème dans l'enseignement de deux APSA collectives différentes (la danse et le handball). L'analyse des contenus propositionnels caractéristiques des règles d'action montre que ceux-ci sont spécifiques à chaque activité et à leur logique interne, cependant, malgré la différence de nature de l'activité, l'aspect collectif et social est valorisé par les élèves dans ces deux activités coopératives. L'analyse des dynamiques interactives montre des différences dans le sens de co-construction au handball et de tutorat spontané en danse.

Mots clés: règles d'action, interlocution, co-construction, comparatisme, socioconstructivisme.

Peer Learning in Physical Education: a comparative analysis of handball and dance dyadic verbal interactions

\section{Summary}

This study is based on an integrative approach to post-Piagetian work (Muller-Mirza and Perret-Clermont, 2008) and Vygotskian works for research on school learning (Brossard and Fijalkow, 1998, Vergnaud, 2002). The main is to consider the construction of specific and transversal skills in P.E. by studying the language role as a conceptual tool. We situate our 


\section{eJRIEPS spécial 2 (juillet 2019)}

work in a comparative perspective by studying students peer dyadic verbal interactions in a french 6th grade in two different collective activities teaching (dance and handball). The analysis of the characteristic propositional contents of the action rules show that they are specific to each activity and their internal logic, however, whereas difference in the activity nature, the collective and social aspect is valued by the students in these two cooperative activities. The analysis of interactive dynamics shows differences in the sense of coconstruction in handball and spontaneous tutoring in dance.

Key words : action rules, interlocution, co-construction, comparatism, socio-constructivism

\section{Introduction}

Cette recherche se situe dans le prolongement des travaux de Darnis \& Lafont $(2008,2013)$ sur l'intérêt des interactions verbales paritaires, en EPS, pour la co-construction de compétences en sport collectif. De plus elle se situe également dans la lignée des travaux de D’Arripe-Longueville, Fleurance \& Winnykamen (1995), pour le développement de compétences motrices individuelles (salto avant et virage brasse) en dyades. Ces études antérieures ont été réalisées en contexte de " quasi expérimentation ".

Nous situons notre travail d'une part en contexte écologique de classe et d'autre part dans une perspective comparatiste en étudiant les interactions verbales paritaires des élèves dans deux APSA différentes (de deux champs d'apprentissage différents). Ces éléments fondent notre originalité au regard des travaux de recherche dans le champ des travaux socioconstructivistes en EPS.

\section{Revue de littérature :}

\subsection{Travail en dyades en EPS}

Les travaux de D'Arripe-Longueville et al. $(1995,2000)$ mettent en évidence les effets du niveau de compétence d'un pair modèle sur la motivation et la performance de sujets novices. Ces travaux montrent que la dissymétrie forte de compétence engendre des effets plus favorables sur les expectations de performance et les progrès des observateurs. Cependant, il n'y aurait pas d'effet dû à l'écart de compétence sur la persévérance. En ce qui concerne les différences liées au genre, l'auteur trouve une supériorité des garçons sur les filles en ce qui concerne les expectations du tutoré, la persévérance et une valorisation 


\section{eJRIEPS spécial 2 (juillet 2019)}

des buts de comparaison sociale et de performance. Par contre les filles ont une prédominance pour les buts auto-référés. Legrain, D’Arripe-Longueville \& Gernigon (2003) mettent également en évidence un gain pour le tuteur dans une relation dyadique d'apprentissage moteur. Les auteurs mettent en place une situation dyadique asymétrique pour un apprentissage en boxe française. Les tuteurs sont experts dans l'activité alors que les tutorés sont novices. Les résultats montrent un gain plus important que l'entraînement individuel, à la fois pour les tutorés et pour les tuteurs.

Par ailleurs, le travail d'analyse des interactions de D'Arripe-Longueville (1998) met en évidence l'existence de trois modalités interactives, inspirées du modèle pluridimensionnel des acquisitions (Beaudichon, Verba \& Winnykamen, 1988) :

- Les conduites de tutelle seraient plutôt caractéristiques des dyades dissymétriques du point de vue de la compétence dans la tâche. Cependant D’Arripe-Longueville (1998) souligne l'importance du statut social et scolaire des pairs en interaction. Dans certains cas, la dissymétrie « objective », liée à la différence de niveau scolaire prend le pas sur la dissymétrie de compétence dans la tâche. On a alors affaire à une « tutelle inversée » ou à une dissymétrie croisée.

- Les conduites de coopération : caractéristiques de dyades symétriques, principalement adoptées par les filles, valorisant les comportements pro-sociaux¹.

- Les conduites parallèles ou de non co-élaboration seraient principalement adoptées par les garçons.

D'Arripe-Longueville (1998) montre également que d'autres facteurs peuvent être responsables de mécanismes interactifs. II s'agit du statut social, du statut scolaire, de la personnalité, du degré de relation amicale, etc... Quelques conduites de confrontation interindividuelles ont été notées mais elles concernent des séquences isolées et de courtes durées. II convient de préciser que dans cette étude, les participants n'ont pas l'obligation d'interagir. La phase d'entraînement est laissée à leur libre choix, ils peuvent profiter du partenaire pour s'entraider ou non.

Lafont et Winnykamen (1999), pour leur part, situent l'analyse des interrelations dans le cadre de la classe et du cours d'EPS, ce qui confère à cette approche une dimension écologique. En s'appuyant sur les travaux de Slavin (1983), de Johnson \& Col. (1981) et de Stanne, Johnson \& Johnson (2000), elles montrent que les situations de coopération apparaissent plus bénéfiques que celles de compétition et de travail individuel. La structure

\footnotetext{
${ }^{1}$ visent à aider, soutenir, réconforter, passent par l'empathie, puis le don, le partage et la coopération, dans le souci de maintenir des relations amicales.
} 


\section{eJRIEPS spécial 2 (juillet 2019)}

même des jeux et sports collectifs, dans leur logique interne, entraîne une coopération entre les membres du groupe formé par l'équipe et une opposition, un affrontement entre les équipes. La situation de jeu est donc simultanément coopérative et compétitive. Ceci plaide en faveur des tâches coopératives, particulièrement en EPS. Pour Lafont, Proères \& Vallet (2007), Lafont, Proères, Burvingt, Menaut \& Poitreau, (1999) « coopérer c'est agir ensemble pour atteindre un but commun, mais coopérer c'est aussi s'entraider, observer, conseiller ses pairs, construire ensemble un projet d'action ". Or, dans les A.P.S., coopération et compétition sont souvent liées, en particulier dans le domaine des sports collectifs. En effet, la logique interne même des jeux et sports collectifs fait apparaître des situations de coopération intra-groupes avec compétition inter-groupes (Lafont \& Winnykamen, 1999).

\subsection{La question de la nature de l'APSA support}

Les travaux de D'Arripe-Longueville (1999) montrent l'intérêt d'un travail en dyades pour le salto avant en gymnastique, puis pour le virage brasse (D'Arripe-Longueville, 2002) en natation. Le travail de Legrain (2003), porte sur l'effet tuteur en boxe française. Darnis (2004) développe les effets des interactions paritaires pour l'apprentissage au choix tactique au hand-ball en dyades symétriques d'une part et, d'autre part, elle montre l'effet de la dissymétrie de compétence pour l'apprentissage au choix tactique au hand-ball. Mascret (2008) prouve l'effet de la coopération du tutorat pour l'apprentissage du badminton en milieu difficile. Ensergueix (2010) explique le rôle du tutorat réciproque et de l'entraînement du tuteur en tennis de table. Enfin, Darnis \& Lafont (2016) montrent le rôle du langage pour la construction de règles en jeux collectifs pour des élèves de 7-8 ans, scolarisés dans le premier degré. Rivière \& Lafont (2014) répondent à la question de l'inclusion en classe ordinaire d'EPS grâce à un dispositif d'apprentissage coopératif d'élèves présentant un handicap cognitif scolarisés en Unité Locale d'Inclusion Scolaire en lutte.

Dans le cadre d'acquisitions d'habiletés motrices morphocinétiques (tâches gymniques aux différents agrès), Legrain \& Heuzé (2005) comparent deux conditions d'apprentissage en groupe : une condition de tutorat réciproque entre pairs organisé par l'enseignant et une condition d'apprentissage coopératif non structuré. De plus, Lafont $(1994,2003)$ montre l'intérêt du développement d'une posture «pluri-procédure » dans la trajectoire professionnelle des futurs et néo-enseignants d'EPS en étudiant les jugements d'efficacité émis à l'égard des différentes procédures pédagogiques en fonction de la nature des habiletés à enseigner. Les résultats montrent un rôle structurant de la formation initiale pour 


\section{eJRIEPS spécial 2 (juillet 2019)}

développer une attitude plurielle et flexible chez les futurs enseignants, avec des procédures de guidage adaptées à la nature des habiletés enseignées.

Les propositions pédagogiques conjointement élaborées par le chercheur et le professeur d'EPS dans notre étude sont largement inspirées des travaux de Dyson (2002) et de Darnis et Lafont (2013), dans une approche intégrative de l'apprentissage coopératif, des interactions sociales appliquées aux apprentissages moteurs et de la didactique comparatiste (Rivière 2012).

Les questions de recherche portent sur la nature des interactions dyadiques selon l'APSA enseignée dans une situation socioconstructiviste en EPS

\section{Questions de recherche}

Dans la continuité des recherches antérieures sur le travail des élèves en dyades, cette recherche vise à identifier des éléments de généricités et de spécificités dans les interactions dyadiques paritaires concernant les activités danse et handball en milieu scolaire. En effet, si l'intérêt des interactions verbales pour l'acquisition de compétences motrices a été largement démontré (D’Arripe-Longueville et al. (1995, 2000) ; Legrain, D’Arripe-Longueville \& Gernigon ,2003; Darnis \& Lafont 2008, 2013 ; Mascret 2008 ; Ensergueix 2010) une étude comparatiste nous semble intéressante pour approfondir ce courant de recherches en STAPS. Ainsi, nous posons les questions suivantes :

- Les contenus propositionnels sont-ils spécifiques des problèmes posés par l'APSA enseignée?

- Les dynamiques interactives sont-elles spécifiques à l'activité enseignée ou dépendent-elles de la nature de la dyade?

- L'aspect collectif des deux situations génère-t-il une centration des interactants sur la coopération entre les partenaires de la dyade?

\section{Cadre théorique}

L'approche théorique se situe au croisement de deux approches: didactique et socioconstructiviste. L'exploitation de ces deux orientations est originale dans la mesure où elle permet de cerner les savoirs en jeu dans les interactions sociales.

\subsection{Perspective comparatiste}

Dans le cadre d'une perspective comparatiste, Rivière (2012) utilise une démarche méthodologique comparatiste des phénomènes interactionnels et didactiques de la classe. 


\section{eJRIEPS spécial 2 (juillet 2019)}

Plusieurs dimensions spécifiques de l'interaction en contexte didactique doivent être considérées. D'une part, le fait que les objets de savoir et les interactions se configurent mutuellement et d'autre part, le fait que l'intersubjectivité et le développement de l'individu sont pris en considération. Par exemple, du point de vue de la Théorie de l'Action Conjointe (TAC), Santini \& Sensevy (2010) décrivent comment, dans la classe, les élèves sont susceptibles de s'approprier un certain type de culture scientifique comme un système d'usage des savoirs géologiques en jeu. Selon eux, décrire les interactions didactiques (définies comme le jeu de l'enseignant sur le jeu de l'élève et réciproquement dans une régulation de l'activité des élèves) observées comme participant d'un jeu d'apprentissage permet d'expliciter l'ici et maintenant de l'action didactique, ses moyens et ses enjeux.

En EPS, comme dans toute autre discipline d'enseignement, il y a certainement un « désir didactique » (Cicurel, 2011, p. 176) qui mobilise les enseignants (parfois, et pour certains d'entre eux tout au moins). Ce désir n'est pas nécessairement partagé par (tous) les élèves... ou pas toujours orienté vers les mêmes objets. Toute situation d'apprentissage est donc aussi traversée par des tensions, des malentendus, des décalages entre ce qui était prévu et ce qui est réalisé, et ceci à différents niveaux : entre ce que l'enseignant a prévu et ce qu'il a réalisé, entre ce qui est prévu par l'enseignant et réalisé par les apprenants, entre ce qui est prévu par le dispositif et réalisé par l'enseignant. Ces décalages fondent, entre autres, des ruptures de contrat didactique.

Mirza \& Perret-Clermont (2008) développent l'idée principale que ces tensions, ces décalages, ne sont pas seulement fréquents, mais constitutifs d'une activité d'apprentissage. Ils peuvent être considérés comme des lieux potentiels de développement, de créativité et d'apprentissage en adoptant une approche socioculturelle qui examine notamment les processus d'interprétation et de construction de sens de la part des acteurs en présence.

En EPS, pour l'acquisition des habiletés motrices, Lafont (2014), dans une approche pluridimensionnelle des acquisitions, distingue l'intervention directe ou indirecte de l'enseignant au sein du groupe classe comme étant plurielle. Pour elle, quand le professeur d'EPS observe ses élèves, les guide, les incite à s'engager, donne des feedbacks, des démonstrations partielles des règles d'action et s'assure que la pratique se réalise en toute sécurité, son intervention est alors directe. Par contre, lorsque l'enseignant fait des choix de formes de regroupement (taille, genre, homogénéité, affinité), attribue à ses élèves des rôles sociaux, anime le travail du groupe et prépare ses élèves à interagir, il intervient alors 


\section{eJRIEPS spécial 2 (juillet 2019)}

indirectement en sollicitant des procédures plurielles: une démarche de recherche autonome de solutions au plan individuel et collectif, des échanges verbaux de type coconstruction, de l'imitation et de la co-observation.

Nous faisons l'hypothèse que, dans une démarche indirecte d'enseignement, mettre les élèves en interactions dyadiques favorise la dévolution didactique. Nous définirons la dévolution comme l'action de l'enseignant consistant à déléguer un certain nombre de tâches à l'élève dans la situation d'enseignement. Celle-ci permet à l'élève de se prendre en charge, d'être responsable de son apprentissage et se traduit chez lui par l'acceptation de rôles sociaux et le développement de compétences méthodologiques sur son propre apprentissage allant dans le sens de compétences métacognitives. La dévolution est un processus par lequel l'enseignant cherche à ce que l'action de l'élève ne soit produite et justifiée que par les nécessités du milieu et par ses connaissances, et non par l'interprétation des procédés didactiques du professeur. Elle consiste, non seulement, à proposer à l'élève une situation qui doit susciter chez lui une activité non convenue, mais aussi à contribuer à sa responsabilisation dans l'obtention du résultat proposé, afin qu'il accepte l'idée que la solution ne dépend que de l'exercice des connaissances qu'il possède déjà (Brousseau \& Balacheff, 1998).

\subsection{Conception socioconstructiviste de l'apprentissage en EPS}

Les travaux réalisés dans le cadre de la psychologie sociale des apprentissages (Doise et Mugny, 1997) et leur application dans le champ des acquisitions en EPS (Darnis \& Lafont 2016; Darnis-Paraboschi, Lafont \& Menault 2006) démontrent l'intérêt d'apprentissages dyadiques et coopératifs pour l'acquisition d'habiletés décisionnelles en sports collectifs dans un contexte de quasi-expérimentation. D'Arripe-Longueville, Fleurance \& Winnykamen (1995) montrent l'intérêt des apprentissages dyadiques pour l'acquisition du salto avant en gymnastique puis du virage brasse.

\subsection{Rôle du langage et de la conceptualisation}

Pour Vygotsky (1985/1934), le langage est un outil ou un instrument de la pensée et le développement d'opérations cognitives nécessite une instrumentalisation dans laquelle le langage joue un rôle important. Les activités langagières ont une double fonction de communication et de représentation, en tant que conceptualisation de l'action.

Vygotsky prétend que les interactions sociales sont primordiales pour un apprentissage dans la Zone Proximale de Développement (ZPD). Dans son sens originel, la ZPD désigne 


\section{eJRIEPS spécial 2 (juillet 2019)}

l'écart entre ce que l'enfant est capable de réaliser seul et ce qu'il peut faire avec l'aide d'un adulte ou d'un pair plus avancé que lui. L'origine sociale de la pensée semble être une caractéristique centrale de la théorie de Vygotsky.

Quant au concept de Conflit Socio Cognitif (CSC) il prolonge et s'éloigne de la conception intracognitive et individualiste du développement de Piaget \& Inhelder (1951). En effet, leurs travaux ont été prolongés par le courant européen de psychologie sociale du développement engageant l'élève dans une dimension socioconstructiviste. II s'agit d'un courant appelé : «l'école de Genève »(Doise \& Mugny,1997 ; Perret-Clermont \& Nicolet 1988), courant influencé par les apports de Vygotsky $(1934,1985)$ dans une approche socio-historique, ainsi que par Bruner (1983). Si Perret-Clermont et Nicolet (1988) ont initié le concept de conflit sociocognitif, leurs travaux montrent qu'en travaillant à plusieurs (en dyades) les enfants atteignent plus rapidement les compétences nécessaires pour accéder au stade de développement concret décrit par Piaget (1976). Ces travaux sont largement repris par Doise \& Mugny (1997) pour faire accéder les apprenants au stade «formel ». En effet, Doise \& Mugny (1997) prolongent les travaux de Piaget \& Vygotsky. Ils présentent les interactions entre pairs comme source de développement cognitif à condition qu'elles suscitent des conflits sociocognitifs. Selon ces deux auteurs, l'interaction sociale est constructive dans la mesure où elle introduit une confrontation entre les conceptions divergentes. Un premier déséquilibre interindividuel apparaît au sein du groupe puisque chaque élève est confronté à des points de vue divergents. II prend ainsi conscience de sa propre pensée par rapport à celle des autres. Ce qui provoque un deuxième déséquilibre de nature intra-individuelle : l'apprenant est amené à reconsidérer, en même temps, ses propres représentations et celles des autres pour reconstruire un nouveau savoir. Le narratif devient, dans cette perspective, un moyen de "penser notre propre pensée" (Bruner, 2000) et renvoie à la compréhension de sa propre pensée ainsi que celle d'autrui.

De leur côté, Gilly, Fraisse \& Roux (1988) (école d'Aix) montrent que les modes de coopération active dans une interaction dyadique sont de quatre types. Ces modes de coopération sont appelés des dynamiques interactives :

- La co-élaboration acquiescante : un des sujets élabore seul une solution et le partenaire ratifie.

- La co-construction ou co-élaboration sans désaccord : l'action de l'un est reprise ou poursuivie par l'autre, le premier prenant à nouveau le relais et ainsi de suite.

- La confrontation contradictoire sans argumentation : confrontation avec désaccords non argumentés. 


\section{eJRIEPS spécial 2 (juillet 2019)}

- La confrontation contradictoire avec argumentation : les confrontations contradictoires se définissent par la présence de désaccords argumentés et/ou d'une autre proposition de procédure. Cette dynamique donne lieu à un Conflit Socio Cognitif (CSC).

C'est à partir de ce cadre théorique des dynamiques interactives que nous analyserons entre autres les interactions entre élèves.

Pour beaucoup d'auteurs, il faut qu'il y ait CSC pour qu'il y ait progrès cognitif. Or il est très difficile et rare d'obtenir des CSC, d'une part parce qu'une situation problème est une condition du CSC et parce que les interactants doivent apporter des solutions différentes à cette situation problème. La plupart du temps il y a accord sur la solution trouvée.

Dans ces conditions, la situation de travail à deux dans la mise en place d'un fonctionnement précis peut se montrer plus bénéfique que la situation de travail individuel à condition de tenir compte : d'une part, du niveau initial des sujets, celui-ci devant se situer à un moment "critique » par rapport à une acquisition. Ce concept rejoint celui de «zone de développement proximale » de Vygotsky (1985b/1934) ; d'autre part, de la dynamique socio-cognitive de la dyade (dynamique interactive). Enfin, l'efficacité du travail en dyade dépend du fonctionnement cognitif suscité par la situation de recherche.

Fraisse (1987) met en évidence que ce sont les désaccords manifestés par le partenaire qui permettent une modification du fonctionnement cognitif du sujet. C'est grâce à la perturbation de l'action ou du système de pensée produite chez le sujet par le partenaire, que celui-ci améliore son niveau cognitif. La fonction perturbatrice du partenaire peut se manifester dans le désaccord et l'argumentation mais également en ouvrant le champ des possibles, particulièrement dans une situation de sport collectif. En effet, il n'y a pas une solution unique mais des solutions possibles en fonction du déroulement de la situation. Pour Fraisse (1987), le partenaire peut avoir différentes fonctions dans l'interaction :

- Une fonction d'activation : le partenaire crée une motivation supplémentaire, permet de dynamogénéiser le travail collectif (Piaget, 1976) et d'intensifier la mobilisation cognitive : (Gilly \& Roux, 1984). D'autre part, le partenaire ouvre le champ des possibles et peut apporter des suggestions nouvelles.

- Une fonction de contrôle et de mémoire.

- Une fonction perturbatrice (contradiction ou non). II peut y avoir contradiction du partenaire ou simple remise en question par le partenaire qui entraîne un obstacle au fonctionnement cognitif du sujet.

- Une fonction d'explicitation-élucidation. 


\section{eJRIEPS spécial 2 (juillet 2019)}

«Le dépassement du conflit socio-cognitif par co-élaboration d'une réponse nouvelle correcte par les sujets en interaction nous paraît être une des possibilités de progrès, certainement la plus efficace » (Fraisse, 1987, p. 952). A l'éclairage de ces travaux, nous nous attendons à une plus grande efficacité des dyades interagissant sur un mode de coconstruction avec des désaccords argumentés ou non, dans lesquelles la fonction perturbatrice du partenaire aura effectivement permis d'ouvrir le champ des possibles et d'envisager plusieurs solutions autant au handball qu'en danse. Le développement de compétences tactiques au handball et de création en danse dans l'interaction sociale devrait être d'autant plus important que les interactions auront donné lieu à une opposition de point de vue ou à des explicitations des solutions envisagées, que l'opposition ou le débat d'idées ait donné lieu à un accord ou non. Dans tous les cas la confrontation de point de vue permet d'ouvrir le champ des possibles et donc engage le sujet vers une attitude réflexive et ouverte, propice aux apprentissages décisionnels. Si le rôle perturbateur du partenaire semble un élément important dans la construction de nouvelles compétences, la situation doit également donner lieu à une perturbation propice aux apprentissages.

Dans ce sens, Carugati (1988) établit une différence au niveau de la nature de la tâche. Pour des tâches fermées, la dynamique conflictuelle sera explicite. La régulation du conflit sera de nature relationnelle ou de nature sociocognitive. Par contre pour une tâche ouverte, ce qui est le cas dans notre propre recherche, tant en danse qu'au handball, l'auteur pense qu'une véritable dynamique interactive pourra avoir lieu, dynamique de résolution de problème avec choix et mise en relation de données pertinentes. II semblerait donc que les tâches ouvertes soient plus propices au conflit de communication que les tâches fermées. A l'éclairage de ces travaux, il semble que la logique interne des situations de sports collectifs, mais aussi les tâches de création de groupe dans les activités à visée expressive et artistique offrent un terrain favorable pour proposer des tâches ouvertes propices à la confrontation de point de vue dans l'interaction dyadique. Encore faut-il que le contexte social soit favorable à la co-construction et à la co-opération.

Aux quatre processus sociocognitifs de dynamiques interactives décrits par Gilly, Fraisse \& Roux (1988), Sorsana (1999) ajoute l'existence de conduites parallèles ou conduites de non co-élaboration. En effet, dans le cas d'interactions dyadiques pour la co-résolution d'une situation problème en sport collectif, il est possible que soit les verbalisations ne portent pas sur la tâche, soit que chaque sujet réponde sans tenir compte du point de vue de son partenaire. Dans ces deux cas il est possible que la dynamique de la dyade ne donne pas 


\section{eJRIEPS spécial 2 (juillet 2019)}

lieu à des conduites de co-résolution. Afin de décrire les processus interactifs, nous nous appuierons sur les travaux de l'analyse interlocutoire et conversationnelle.

Gilly, Roux \& Trognon (1999) proposent l'observation des médiations sémiotiques pour comprendre les mécanismes interactifs propices au progrès dans l'interaction. Les interactions entre partenaires sont finalisées par la réalisation de tâches imposées de l'extérieur ou que les sujets se fixent eux-mêmes de façon plus ou moins implicite. Le modèle d'analyse de la tâche et/ou du fonctionnement de résolution permet d'analyser les comportements verbaux et non verbaux des participants du point de vue des objectifs visés. Les interactions sont toujours inscrites dans un contexte dont les niveaux fonctionnels sont à prendre en compte pour guider l'analyse. II convient tout d'abord de faire une analyse de la tâche en termes de contexte situationnel, contexte interactionnel et contexte interdiscursif. Ils constituent trois niveaux fonctionnels du contexte interactif. Le contexte situationnel définit le contrat de communication, les conditions institutionnelles, temporelles et matérielles de la situation d'interactions verbales. Ce contexte, encore appelé métacontrat, permet de situer les régularités, les routines ou les formats de communication. Le contexte interactionnel ou contexte interdiscursif est « logé dans le premier » et concerne les interactions proprement dites, c'est à dire les influences réciproques, les signes. II représente le cadre de l'analyse interlocutoire. Le contexte interdiscursif est constitutif du contexte interactionnel. Ce sont les aspects langagiers proprement dits. Ils se réfèrent au contexte de la langue. L'analyse conversationnelle décrite par Trognon (1999) a été conçue comme une démarche d'investigation des structures normatives des raisonnements impliqués dans la compréhension et la production de l'intelligibilité du déroulement de l'interaction. Elle étudie l'organisation séquentielle de l'interaction et du raisonnement qui I'habite. La conversation constitue une matrice de la pensée. La filiation vygotskienne est fortement marquée. Les progrès observés à l'occasion d'interactions sociales sont, de ce point de vue, explicables par une transformation de fonctionnements inter-individuels en fonctionnements intra-individuels (Gilly \& Deblieux, 1999). La dynamique de l'interlocution produit conjointement du social et du cognitif. La micro-analyse des interactions sémiotiques de la communication tente de comprendre, s'appuyant sur la théorie des actes de langage, par quels processus précis le déroulement effectif d'une interaction de co-résolution peut être ou non bénéfique. Dans la théorie des actes de langage, chaque illocution est considérée comme un acte social composé d'une force illocutoire $(F)$ appliquée à un contenu propositionnel $(p)$. La fonction $F(p)$ caractérise chaque acte illocutoire. La distinction entre la force et le contenu propositionnel est fondamentale. La force permet de 


\section{eJRIEPS spécial 2 (juillet 2019)}

voir comment, par leurs comportements langagiers, les sujets gèrent les aspects socio relationnels et actionnels des échanges. Le contenu propositionnel renvoie aux aspects cognitifs représentationnels des échanges. Chaque acte illocutoire est donc analysé du point de vue de sa force et de son contenu propositionnel. Dans l'analyse des interactions verbales que nous proposerons outre l'analyse de la dynamique interactive de la dyade, l'analyse du contenu propositionnel devrait permettre de comprendre les connaissances en jeu dans l'interaction (autant au hand-ball qu'en danse). La force, associée au contenu propositionnel, devrait étayer celui-ci afin de convaincre le partenaire de la véracité et de l'efficacité de la solution envisagée. Cependant, c'est le contenu propositionnel lui-même qui se rapporte au plus près de la formulation de règles d'action. Nous nous attendons à ce que le contenu propositionnel soit relatif au projet d'action et permette, par la formulation de règles d'actions, une amélioration des compétences tactiques en jeu.

En ce qui concerne la construction de compétences en EPS, il semblerait que le langage puisse avoir un rôle d'outil conceptuel. En effet, pour Vergnaud (2002), C'est avant tout dans la conceptualisation, dans la prise de conscience que se construisent les règles d'action constitutives de schèmes plus élaborés et généralisables. Nous proposons une démarche d'apprentissage visant à développer la compréhension et la généralisation de schèmes grâce aux interactions verbales dyadiques dans lesquelles la conceptualisation ou la prise de conscience repose sur des phénomènes métacognitifs.

\subsection{Le rôle des métaconnaissances}

Les métaconnaissances de compétences sont définies comme étant le savoir de l'opérateur sur les caractéristiques de son propre fonctionnement cognitif. "La métacognition se rapporte à la connaissance qu'on a de ses propres processus cognitifs » (Flavell 1979, p. 906). Il s'agit pour l'élève de comprendre comment il fait pour mémoriser, pour apprendre, pour résoudre, pour réaliser une tâche complexe. Ce processus métacognitif rend compte d'un passage du « réussir » au " comprendre ». Ainsi, pour Flavell (1979) la métacognition concerne trois aspects : les personnes, les tâches et les stratégies.

Pour les personnes, il s'agit de toutes les connaissances concernant le fonctionnement cognitif de soi et des autres. II s'agit des connaissances que les personnes possèdent sur leurs propres compétences cognitives.

Pour les tâches, il s'agit d'une part de la nature des informations à traiter (schématisation, sous-paragraphes, tableaux, graphiques ou textes explicatifs) et d'autre part de la nature des exigences de la tâche (difficulté plus ou moins grande perçue par le sujet). 


\section{eJRIEPS spécial 2 (juillet 2019)}

Enfin, pour les stratégies, il s'agit des connaissances mises en œuvre pour résoudre la tâche : ce qu'il faut faire pour faire.

Par ailleurs, Vergnaud (1996) relève différentes formes de savoirs intervenant dans le développement de compétences professionnelles. II s'agit des savoirs d'action impliqués dans la maîtrise de situations complexes et intellectuelles : d'une part, les compétences perceptuo-gestuelles de maîtrise, d'autre part de la forme linguistique et symbolique des connaissances et plus généralement de la communication entre individus à propos de situations à maîtriser, qu'il nomme le « débat de points de vue » et pour laquelle la conceptualisation est indispensable à la maîtrise de la forme et enfin, des compétences sociales qui interviennent dans l'interaction avec autrui dans l'interprétation des signes verbaux et non verbaux, dans la communication.

Rappelons que pour Vergnaud (1996), un savoir d'action ne peut se construire sans la conceptualisation. Le concept de schème donc pour lui la clé de voûte entre savoirs d'action et savoirs théoriques. II est au cœur de la conceptualisation. Le schème est une totalité dynamique fonctionnelle. II correspond à un but identifiable et représente une unité dynamique et temporelle identifiable. Le schème est également une organisation invariante de la conduite pour une classe donnée de situations. II est donc un universel en ce sens qu'il renvoie à une classe. Pour Vergnaud (1998), il y a intégration des schèmes. La formation des connaissances opératoires consiste à la fois dans des gestes et des pratiques difficiles à acquérir et dans des conceptualisations subtiles. Ainsi, un schème est formé de plusieurs catégories d'éléments, tous indispensables : des buts, une anticipation, des règles d'action, des possibilités d'inférence en situation et des invariants opératoires.

Au plan pragmatique de l'intervention en sports collectifs, les travaux sur le débat d'idée se sont dirigés vers deux pistes pédagogiques, proposant des mises en œuvre du débat d'idées à des moments différents du processus d'apprentissage. D'un côté, Gréhaigne \& Godbout (1995), proposent une opérationnalisation en trois temps : un premier temps de jeu (temps d'action où les élèves sont en action) un second temps pour la co-observation et la co-évaluation; un troisième temps pour le « débat d'idées ». Dans cette optique le temps de débat et d'échanges verbaux repose sur la définition de rôles sociaux (observateur) et d'outils (fiches, grilles d'observation) servant de support aux coconstructions de règles d'action. D'un autre côté, le modèle anglosaxon du Teaching Games For Understanding (TGFU) a été développé initialement par Bunker \& Thorpe (1982) et propose dans les années 80 une alternative à l'enseignement techniciste des sports 


\section{eJRIEPS spécial 2 (juillet 2019)}

collectifs à l'école. A partir de situations de jeu à effectif réduit, les apprenants sont invités à " apprécier le jeu » dans un but de conceptualisation, puis à prendre conscience des aspects tactiques importants pour résoudre les problèmes posés par la confrontation collective. Ensuite l'enseignant guide l'apprenant vers l'identification de règles visant le "quoi faire ", mais aussi le " comment le faire ", c'est-à-dire " ce qu'il y a à faire pour faire ». Cette perspective est synthétisée par Grehaigne, Godbout et Mahut (1995). Pour favoriser la co-émergence de concepts pragmatiques utiles au développement de compétences en sports collectifs, nous prenons également appui sur les travaux de Chang et al. (2006). Ils proposent de mettre en place des phases d'échanges, de débats d'idées au sein des équipes entre les séquences de jeu (il s'agit de basket avec des élèves de 11 ans). Ils mettent en évidence les phénomènes d'extraction des règles de l'action efficace dans un espace dialogique et partagé de la cognition au sein du groupe.

Par ailleurs, dans la double perspective de l'apprentissage coopératif et des formats pédagogiques du TGFU, Dyson (2002) développe des propositions pour l'apprentissage coopératif en sports collectifs à l'école élémentaire. Ses propositions reposent sur l'utilisation de rôles sociaux (observateurs, coachs) et de débats en petits groupes pour favoriser les apprentissages en jeux collectifs chez des enfants jeunes et novices.

Les propositions pédagogiques conjointement élaborées par le chercheur et le professeur d'EPS dans cette étude sont largement inspirées des travaux de Dyson (2002) et de Darnis \& Lafont (2013), dans une approche intégrative de l'apprentissage coopératif, des interactions sociales appliquées aux apprentissages moteurs et de la didactique des sports collectifs (Gréhaigne, 2003).

\section{Méthodologie}

C'est en s'appuyant sur les exigences de la recherche participative que nous avons conjointement élaboré un protocole de recherche action de collaboration entre un chercheur et un professeur d'EPS. D'après Bourassa, Bélair \& Chevalier (2007), la recherche participative doit répondre à six critères : le sujet de recherche doit être vécu comme pertinent pour le chercheur et les praticiens ; la recherche doit examiner une question ou un problème co-construit et co-défini ; elle s'enrichit dans l'intersubjectivité de la collaboration chercheur/praticien à toutes les étapes; elle mène à une théorisation temporaire ; elle entérine un changement effectif de la situation perçue ; et enfin, elle produit des effets d'auto-formation. 


\section{eJRIEPS spécial 2 (juillet 2019)}

\subsection{Contexte et participants}

Pour chaque APSA, le chercheur a formalisé avec l'enseignant une modalité d'enseignement en dyade. Cinq séances d'enseignement (handball et danse) étaient observées pour une même classe de Gème (24 élèves, 12 filles et 12 garçons âgés de 11/12 ans) dans un collège de banlieue bordelaise sans problème particulier.

Ces séances d'enseignement étaient mises en œuvre par une enseignante chevronnée (plus de 5 ans d'expérience dans l'enseignement de l'EPS, tutrice de stagiaires en formation initiale et membre du groupe de formateurs de l'ESPE d'Aquitaine pour l'écrit 2 du CAPEPS), assistée du chercheur, dans une recherche action ou recherche participative.

- En danse le travail en dyade se situait pendant la phase d'exploration à partir d'un travail en duo avec un inducteur favorable à la création de mouvements dansés. II s'agissait de composer un duo à partir d'un inducteur : écrire à partir de lettres réalisées corporellement. Les interactions verbales avaient lieu conjointement au travail de création. Elles étaient recueillies à l'aide d'un camescope en plan serré.

- Au handball, le travail en dyade se situait lors d'une situation d'apprentissage favorable à la prise de décision : un 2 contre 1 sur un demi-terrain avec interactions verbales entre les passages pour se mettre d'accord sur la stratégie (conformément à la recherche de Darnis (2004). L'enseignante demande aux élèves : "Que faut-il faire pour arriver à tirer sans se faire prendre le ballon par le défenseur? ».

\subsection{Procédure de recueil de données}

Les interactions entre élèves étaient analysées lors des séances deux et trois sur une séquence de huit séances dans chaque APSA. Chaque leçon a duré 1 h30 effective, le collège disposant d'installations intra-muros.

Les élèves étaient répartis en dyades légèrement dissymétriques à partir d'une évaluation initiale au handball en séance une. Les mêmes dyades étaient conservées pour le cycle de danse. Au handball, la situation d'apprentissage est une montée de balle à deux attaquants contre un défenseur sur un demi-terrain. Chaque dyade doit alterner un passage avec une phase d'interactions verbales pour se mettre d'accord sur la stratégie. En danse les élèves devaient composer un duo à partir d'un inducteur : utiliser son corps pour former des lettres. Sur les douze dyades constituées, seules cinq dyades pouvaient faire l'objet d'un traitement à cause de l'absentéisme entre les séances. 


\section{eJRIEPS spécial 2 (juillet 2019)}

\section{3. Recueil de données}

Toutes les interactions dyadiques étaient enregistrées (caméra en plan serré). En effet, les données ont été captées d'une part à l'aide d'une caméra fixe en plan large (pour filmer l'intégralité de la séance) et d'autre part, d'une deuxième caméra mobile (en plan serré) portée à l'épaule par le chercheur afin d'enregistrer l'exécution de la tâche de chaque dyade ainsi que les échanges verbaux entre les partenaires après chaque passage. Cette procédure est identique au handball comme en danse.

\subsection{Traitement des données}

Les interactions dyadiques étaient retranscrites in extenso sous forme de verbatim (dont nous rendrons compte sous forme d'extraits dans la partie résultats). Dans un troisième temps ce verbatim était catégorisé d'une part selon les contenus propositionnels au regard de la didactique de chaque APSA, et d'autre part selon la dynamique interactive de la dyade (Gilly, Fraisse et Roux 1988 ; Darnis et Lafont, 2008).

Les catégories d'analyse des contenus propositionnels ont été définies à postériori à partir des catégorisations retenues par Darnis (2004) (technico-tactique : démarcage ; passe ; dribble ; stratégie collective : si... alors... ; placement de joueurs) pour le hand-ball, et de Guerber-Walsh, Leray et Maucouvert, 1997) (temps ; énergie ; espace ; organisation collective) pour la danse.

Par exemple :

- « Je te fais la passe et après je cours devant et tu me la redonnes » est catégorisé comme un contenu propositionnel au handball de type technico-tactique.

- " Moi je fais comme ça » (démonstration) et toi tu enchaînes après à côté et après on le fait ensemble » est catégorisé comme un contenu propositionnel en danse de type espace et organisation collective.

\section{Résultats}

\subsection{Résultats de l'analyse des contenus propositionnels des échanges verbaux dans les dyades cibles.}

L'analyse des contenus propositionnels caractéristiques des règles d'action en lien avec l'activité enseignée est présentée dans le tableau ci-dessous). 


\section{eJRIEPS spécial 2 (juillet 2019)}

Tableau I: Nombre de règles d'action pour chaque catégorie de contenu propositionnel comptabilisé par l'ensemble des dyades cibles.

\begin{tabular}{|l|l|l|l|l|l|l|l|}
\hline & $\begin{array}{l}\text { Compréhension } \\
\text { de la règle } \\
\text { (faute, marché, } \\
\text { zone...) }\end{array}$ & $\begin{array}{l}\text { Justification } \\
\text { technico- } \\
\text { tactique }\end{array}$ & $\begin{array}{l}\text { Stratégie } \\
\text { collective, } \\
\text { organisation } \\
\text { d'équipe, } \\
\text { placement } \\
\text { des joueurs }\end{array}$ & $\begin{array}{l}\text { Gestuel } \\
+\end{array}$ & $\begin{array}{l}\text { Gestuel } \\
\text { espace } \\
\text { et } \\
\text { intension }\end{array}$ & $\begin{array}{l}\text { Organisation } \\
\text { vitesse } \\
\text { collective du } \\
\text { groupe }\end{array}$ & $\begin{array}{l}\text { Intention } \\
+ \\
\text { énergie }\end{array}$ \\
\hline Handball & 1 & 1 & 4 & - & - & - & - \\
\hline Danse & - & - & - & 2 & 1 & 3 & 1 \\
\hline
\end{tabular}

Il est particulièrement remarquable de noter que dans ces deux activités, contrastées au niveau de leur logique interne, mais similaires au niveau du but collectif de la tâche, les interlocutions les plus nombreuses portent sur l'organisation collective du groupe en danse et sur les stratégies collectives, l'organisation d'équipe, le placement des joueurs au handball. Ce résultat montre que lors d'une situation didactique de type socioconstructiviste, les élèves se centrent d'une part sur le problème à résoudre : en ce sens ils co-construisent des compétences spécifiques à l'APSA (technico-tactique au handball ; temps, énergie, espace, en danse), d'autre part, ils se centrent sur l'aspect collectif de la tâche. En ce sens ils construisent des compétences sociales.

Extraits de verbatim (Sabrina et Clara en handball et en danse)

La discussion et l'échange verbal entre les élèves qui forment la dyade montrent la coopération et le travail de groupe afin de résoudre les problèmes rencontrés dans l'exécution de la tâche demandée en handball comme en danse tels qu'en témoignent les extraits de verbatim suivants :

Handball :

- Sabrina : on part du passage d'avant on a discuté avant et on s'est dit qu'on l'attire vers moi et comme la prof a dit il faut mettre la distance entre le défenseur et moi et voilà !

- Clara : au début c'était moi qui devais tirer mais bon vu qu'il est venu directement sur moi il fallait changer de stratégie

Danse : 


\section{eJRIEPS spécial 2 (juillet 2019)}

Sabrina : Je vais faire "gratter", ensuite je vais faire un « $U$ », je vais faire un « $U$ » et on va dire que j'ai creusé, moi j'ai décidé de creuser avec mon coude!

Clara : Be non!

Sabrina : Et pourquoi pas?

Clara : Ce n'est pas creuser.

Sabrina : Et si je te dis que c'est creuser ! pour moi c'est creuser !

Clara : Ah oui !

Sabrina : Regarde bien je vais creuser là ! mon « $U$ » dans l'espace ! on part làdessus ! il y a trois consignes, il y a un trajet à respecter donc avant de venir sur le trajet il faut créer les quatre lettres avec les parties du corps, en thématique! On joue ?

Dans ces extraits de verbatim Sabrina et Clara interagissent mutuellement au handball, dans une co-construction (chacune propose une solution répondant à la précédente pour l'enrichir : " on l'attire vers moi », «il fallait changer de stratégie »). De plus, en prenant en compte la position du défenseur pour adapter la prise de décision : «on l'attire vers moi.../ venu directement sur moi il fallait changer de stratégie ", cet acte de langage démontre la prise en compte d'un algorithme d'attaque afin de réaliser des actions décisives en situation favorable pour faire basculer le rapport de force en sa faveur ou en faveur de son coéquipier qui est caractéristique de la résolution technico-tactique du problème du 2 contre 1 au handball.

En danse, le contenu propositionnel porte sur l'intention créative avec une centration sur l'énergie de la forme gestuelle conformément à l'attente de l'enseignant pour cette tâche. II s'avère que Sabrina participe à l'AS danse et se positionne en tant que tutrice de sa partenaire Clara. L'aspect coopératif entre les deux danseuses se caractérise ici par une forte dissymétrie : dans un premier temps, il y a co-construction entre les partenaires de la dyade mais par la suite Sabrina impose son point de vue dans une co-élaboration acquiesçante. Afin d'affiner ce résultat, nous analysons au niveau microscopique de la dyade les dynamiques interactives.

6. Résultats de l'analyse des dynamiques interactives des échanges verbaux dans les dyades cibles.

Dynamiques interactives : 


\section{eJRIEPS spécial 2 (juillet 2019)}

Tableau II: Nombre de dynamiques interactives comptabilisé pour chaque catégorie pour l'ensemble des dyades cibles

\begin{tabular}{|l|l|l|}
\hline & HB & Danse \\
\hline Co-élaboration acquiesçante & 18 & - \\
\hline Co-construction sans désaccord & 114 & - \\
\hline Tutorat spontané & - & 144 \\
\hline $\begin{array}{l}\text { Co-construction avec désaccord non } \\
\text { argumenté }\end{array}$ & 27 & 54 \\
\hline $\begin{array}{l}\text { Co-construction avec désaccord } \\
\text { argumenté (CSC) }\end{array}$ & - & 53 \\
\hline
\end{tabular}

Dans l'analyse des interactions dyadiques au handball, nous observons une majorité de co-construction sans désaccord. Ce résultat est conforme à ceux de Darnis (2004). Cependant, contrairement à cet auteur, nous n'observons pas de tutorat spontané au handball.

En revanche, en danse, nous observons une proportion importante de tutorats spontanés. Ce phénomène semble spécifique aux dyades féminines dans lesquelles une des deux partenaires prend l'initiative de la construction chorégraphique, cantonnant sa partenaire au rôle de danseuse.

Analyse qualitative des dynamiques interactives dans une dyade :

Tableau III : Extraits de verbatim dans chaque APSA, selon la dynamique interactive.

\begin{tabular}{|c|c|c|c|c|}
\hline & $\mathrm{HB}$ & Extrait HB & Danse & Extrait danse \\
\hline $\begin{array}{l}\text { Co-élaboration } \\
\text { acquiesçante }\end{array}$ & 18 & $\begin{array}{l}\text {-«t'as bien regardé ce qu'il s'est } \\
\text { passé »? } \\
\text {-«Non » } \\
\text { - T'avais les pieds dans la zone } \\
\text { c'est pour ça que l'arbitre a sifflé } \\
\text { une faute! » } \\
\text { - T'as raison j'ai couru vite au } \\
\text { point que je n'ai pas vu la ligne.. » }\end{array}$ & & \\
\hline $\begin{array}{l}\text { Co-construction } \\
\text { sans désaccord }\end{array}$ & 114 & $\begin{array}{l}\text { - On se fait tout le temps prendre } \\
\text { la balle? ? }\end{array}$ & & \\
\hline
\end{tabular}




\begin{tabular}{|c|c|c|c|c|}
\hline & & $\begin{array}{l}\text {-« Normal Angélina on est trop } \\
\text { serré !! » } \\
\text {-«Tout à l'heure je vais avancer } \\
\text { seule sans la balle une fois } \\
\text { arrivée tu me la passes! » } \\
\text { - On verra bien ce que ça } \\
\text { donne » }\end{array}$ & & \\
\hline Tutorat spontané & - & & 144 & $\begin{array}{l}\text {-«On va d'abord sans la } \\
\text { musique! c'est moi qui va } \\
\text { compter décale toi bon le } 1 \\
234 \text { c'est facile le } 56 \text { non } \\
\text { plus! t'arrive à le faire ? } \\
\text { c'est facile enroule ton bras } \\
\text { et le coude tu le mets à } \\
\text { l'intérieur» }\end{array}$ \\
\hline $\begin{array}{l}\text { Co-construction } \\
\text { avec désaccord } \\
\text { non argumenté }\end{array}$ & 27 & $\begin{array}{l}\text {-«Sarah c'est la deuxième fois où } \\
\text { tu nous fais perdre la balle le } \\
\text { prochain passage c'est moi qu } \\
\text { tire! » } \\
-« \text { Non si je me retrouve devant la } \\
\text { zone je tire » }\end{array}$ & 54 & $\begin{array}{l}\text {-«Mona t'en pense quoi ? } \\
\text { Est-ce que tu veux faire ça } \\
\text { (démonstration) à la place? } \\
-« \text { Non merci » }\end{array}$ \\
\hline $\begin{array}{l}\text { Co-construction } \\
\text { avec désaccord } \\
\text { argumenté (CSC) }\end{array}$ & - & & 53 & $\begin{array}{l}\text { - Y a 5mn que tu fais ça } \\
\text { (démonstration)! Elle est } \\
\text { où ta chorégraphie? » } \\
\text {-C'est parce que je veux } \\
\text { changer » } \\
\text {-«Ah oui c'est ça ton } \\
\text { changement?» } \\
\text {-«Beh oui faut que ça } \\
\text { tourne! » }\end{array}$ \\
\hline
\end{tabular}

Dans cette dyade féminine: Mona et Sarah utilisent une forme de tutorat spontané et de co-construction avec désaccord argumenté uniquement en danse, alors que la coélaboration acquiesçante n'est utilisée qu'au handball. Cet extrait révèle une plus grande richesse de dynamiques interactives porteuses de progrès en danse qu'au handball dans ce cas. 


\section{eJRIEPS spécial 2 (juillet 2019)}

\section{Discussion}

Les études dans le champ de la psychologie sociale des apprentissages appliquées aux acquisitions motrices sont nombreuses dans le domaine de la recherche en STAPS (D’Arripe-Longueville, 1998, 2000, 2002) ; Lafont (2002, 2014) ; Darnis (2004) ; Legrain, D’Arripe-Longueville et Gernigon (2003) ; Darnis et Lafont (2008) ; Ensergueix et Lafont (2010) et portent sur des APSA variées (danse, natation, handball, tennis de table, boxe française). Notre originalité porte sur l'aspect comparatiste de cette recherche. En effet l'enseignement en dyades s'est généralisé dans le domaine de l'intervention en EPS.

Par ailleurs, l'ensemble de nos résultats confirment les travaux de Darnis et al. (2006) démontrant l'intérêt du travail en dyades symétriques avec interactions verbales pour la coconstruction des compétences tactiques. Cependant, la présente étude montre que, conformément aux travaux antérieurs dans le domaine des tâches de développement cognitif ou de résolution de problèmes (Doise et Mugny, 1997 ; Howe, 1992), une supériorité de la condition de groupement dissymétrique est observée. Ce résultat est confirmé par la mesure des progrès qui montre que la forme de groupement influence les progrès dans le sens où la condition dissymétrique est plus favorable aux progrès.

\section{Conclusion}

Cette étude plaide en faveur d'un rapport au savoir et à l'interaction sociale dans une didactique socioconstructiviste aussi bien en sport collectif qu'en danse pour une coconstruction de compétences coopératives grâce aux interactions langagières paritaires.

Le rôle des interactions sociales dyadiques, qui a été démontré en EPS par Darnis (2004), Darnis et Lafont (2008) et d'Arippe-Longueville (1999, 2000) en contexte de « quasi expérimentation, repose sur la base des travaux de psychologie sociale du développement pour analyser la relation entre le développement cognitif, les interactions sociales et l'apprentissage de choix tactiques en sports collectifs afin d'étudier les progrès réalisés en dyades symétriques dans une tâche décisionnelle en handball. Les résultats montrent que les sujets bénéficiant de verbalisations en dyades progressent davantage que les sujets sans interactions verbales et que le niveau de développement opératoire influence positivement les progrès réalisés dans cet apprentissage coopératif.

Notre étude s'inscrit dans le prolongement de ces études en contexte écologique de classe. L'analyse des interactions dyadiques montre des généricités et des spécificités selon l'APSA enseignée. Par ailleurs, les interactions didactiques langagières de l'enseignant feront l'objet d'une analyse approfondie complémentaire en prolongement de cette étude. 


\section{Bibliographie}

Arripe-Longueville (d') F. (1998). Contribution à l'identification de processus cognitifs et de mécanismes interactifs impliqués lors de l'acquisition en dyade d'une habileté motrice complexe. Thèse de doctorat non publiée. Université de Paris 10, Paris.

Arripe-Longueville, (d') F., Fleurance, P., \& Winnykamen, F. (1995). Effects of the degree of competence symmetry in the acquisition of a motor skill in a dyad. Journal of Human Movement Studies, 28(6), 255-274.

Arripe-Longueville, (d') F., Gernigon, C., Huet, M. L., Cadopi, M., \& Winnykamen, F. (2002). Peer tutoring in a physical education setting: Influence of tutor skill level on novice learners' motivation and performance. Journal of Teaching in Physical Education, 22(1), 105-123.

Arripe-Longueville, (d') F., Het, M. L., \& Gernigon, C. (2000). Peer relationships and motor skill acquisition: Effects of skill level asymmetry and gender on interactive dynamics and achievement. Journal of Sport \& Exercise Psychology, 22.

Beaudichon, J., Verba, M., \& Winnykamen, F. (1988). Interactions sociales et acquisition de connaissances chez l'enfant : une approche pluridimensionnelle. Revue internationale de psychologie sociale, 1, 129-141.

Bourassa, M., Bélair, L., Chevalier, J., (2007). Les outils de la recherche participative, Education et Francophonie, XXXV (2), 1-11.

Brossard, M., \& Fijalkow, J. (1998). Apprentissage à l'école : Perspectives piagétiennes et vygotskiennnes. Bordeaux : PUB.

Brousseau, G., \& Balacheff, N. (1998). Théorie des situations didactiques : Didactique des mathématiques 1970-1990. (Textes rassemblés et préparés par Nicolas Balacheff, Martin Cooper, Rosamund Sutherland, Virginia Warfield). (pp.470-482). Grenoble : La Pensée Sauvage, Collection Recherches en Didactique des Mathématiques.

Brossard, M. \& Fijalkow, J. (1998). Apprendre à l'école : perspective piagetienne et vygotskiennes. Talence: Presses Universitaires de Bordeaux.

Bruner, J. S. (1983). Savoir faire. Savoir dire. Paris : PUF.

Bruner, J. S. (2000). Piaget et Vygotsky. Célébrons la divergence. In L'esprit piagétien (pp. 237-253). Paris : PUF.

Bunker, D., \& Thorpe, R. (1982). A model for the teaching of games in the secondary school. Bulletin of Physical Education, 10, 9-16. 


\section{eJRIEPS spécial 2 (juillet 2019)}

Chang, C. W., Walian, N., Nachon, M., \& Gréhaigne, J.F. (2006). Pratiques langagières et stratégies d'action: vers une approche semio-constructiviste du basket-ball à Taiwan. Staps, (2), 63-77.

Cicurel, F. (2011). De l'analyse des interactions en classe de langue à l'agir professoral : une recherche entre linguistique interactionnelle, didactique et théories de l'action. Pratiques. Linguistique, littérature, didactique, 149-150, 41-55.

Darnis, F. (2004). Place et rôle du développement cognitif et de l'interaction sociale dans l'acte tactique en jeux et sports collectifs. Thèse de doctorat non publiée. Université de Bordeaux, Bordeaux.

Darnis-Paraboschi, F., Lafont, L., \& Menaut, A. (2006). Interactions sociales en dyades symétriques et dissymétriques dans une situation d'apprentissage au handball. Staps, 3, 25-38.

Darnis, F., \& Lafont, L. (2008). Effets de la dissymétrie de compétence pour un apprentissage coopératif en dyades en Education Physique et Sportive Cahiers Internationaux de Psychologie Sociale, 79, 69-84.

Darnis, F., \& Lafont, L. (2013). Cooperative learning and dyadic interactions: two modes of knowledge construction in socio constructivist settings for team sport teaching, Physical Education and Sport Pedagogy, 20(5), 1-15.

Darnis, F., \& Lafont, L. (2016). Langage et construction de règles en jeux collectifs. Recherche en éducation, 24, 183-197.

Doise, W., \& Mugny, G. (1997). Psychologie sociale et développement cognitif. Paris: A. Colin.

Dyson, B. (2002). The implementation of cooperative learning in an elementary physical education program. Journal of teaching in Physical Education, 22(1), 69-85.

Ensergueix, P. J., \& Lafont, L. (2010). Reciprocal peer tutoring in a physical education setting: influence of peer tutor training and gender on motor performance and self-efficacy outcomes. European Journal of Psychology of Education, 25(2), 222-242.

Flavell, J. H. (1979). Metacognition and cognitive monitoring: A new area of cognitivedevelopmental inquiry. American psychologist, 34(10), 900-906.

Fraisse, J. (1987). Etude du rôle perturbateur du partenaire dans la découverte d'une stratégie cognitive chez des enfants de 11 ans en situation d'interaction sociale. Bulletin de psychologie, tome $\mathrm{XL}, 382,943-952$. 


\section{eJRIEPS spécial 2 (juillet 2019)}

Gilly, M., \& Deblieux, M. (1999). Analyse des médiations langagières en situation dyadique de résumé de récit. In M. Gilly, J.-P. Roux, \& A. Trognon, (Eds.), Apprendre dans l'interaction (pp. 95-120). Nancy : Presses Universitaires de Nancy.

Gilly, M., Fraisse, J., \& Roux, J. P. (1988). Résolution de problèmes en dyades et progrès cognitifs chez des enfants de 11 à 13 ans : dynamiques interactives et mécanismes sociocognitifs. In A. N., Perret-Clermont, \& M. Nicolet, Interagir et connaître. Enjeux et régulations sociales dans le développement cognitif (pp. 60-78). Cousset: Delval,.

Gilly, M., Roux, J. P., \& Trognon, A. (1999). Apprendre dans l'interaction : analyse des médiations sémiotiques. Nancy : Presses universitaires de Nancy.

Gilly, M. \& Roux, J.P. (1984). Efficacité comparée du travail individuel et du travail en interaction socio-cognitive dans l'appropriation et la mise en œuvre de règles de résolution chez des enfants de 11-12 ans. Cahiers de psychologie cognitive, 4, 171-188.

Gréhaigne, J. F. (2003). Vers une didactique constructiviste en sport collectif. In C. AmadeEscot, (Ed.), Didactique de l'éducation physique : État des recherches (pp. 79-102). Paris : Revue EPS.

Gréhaigne, J. F., \& Godbout, P. (1995). Tactical knowledge in team sports from a constructivist and cognitivist perspective. Quest, 47(4), 490-505.

Gréhaigne, J. F., Godbout, P., \& Mahut, N. (1999). L'enseignement des jeux par la compréhension : une revue de question. STAPS,. 48, 81-93.

Guerber Walsh, N., Leray, C., Maucouvert, A. (1997). Danse. Paris : Éd. Revue EPS, coll. de l'école aux associations.

Howe, K. R. (1992). Getting over the quantitative-qualitative debate. American Journal of Education, 100(2), 236-256.

Johnson, D. W., Maruyama, G., Johnson, R., Nelson, D., \& Skon, L. (1981). Effects of cooperative, competitive, and individualistic goal structures on achievement: A metaanalysis. Psychological bulletin, 89(1), 47.

Johnson, D. W., Johnson, R. T., \& Stanne, M. B. (2000). Cooperative learning methods: A meta-analysis. Minneapolis University of Minnesota.

Lafont, L. (1994). Modalités sociales d'acquisition d'habiletés motrices complexes: rôles de la démonstration explicitée et d'autres procédures de guidage selon la nature des habiletés. Thèse de doctorat non publiée. Université de Paris 5 , Paris.

Lafont, L., \& Winnykamen, F. (1999). Co-operation and competition in children and adolescents. In Y. Vauden Ausweele, F. Bakker, S. Biddle, M. Durand, \& R. Seiler (Eds),. Psychology for Physical Educators (pp. 379-404). Champaign, IL : Human Kinetics. 


\section{eJRIEPS spécial 2 (juillet 2019)}

Lafont L., Proeres M., Burvingt F., Menaut A. \& Poitreau D. (1999). Les situations de coopération en EPS, identifier les effets de l'intervention en motricité humaine. Actes du colloque AFAPS-EDPM (CD-Rom). Louvain La Neuve.

Lafont, L. (2002). Efficacité comparée de la démonstration explicitée et de l'imitationmodélisation interactive pour l'acquisition d'une séquence dansée chez des adolescentes de 12 à 15 ans. Staps, 2, 69-79.

Lafont, L. (2003). Etude des relations entre les caractéristiques sportives des étudiants en STAPS et leur jugement à l'égard de l'efficacité des différentes procédures d'enseignement. Sciences et motricité, 48, 9-28.

Lafont, L., Proeres, M., \& Vallet, C. (2007). Cooperative group learning in a team game: role of verbal exchanges among peers. Social Psychology of education, 10(1), 93-113.

Lafont, L. (2014). Approche pluridimensionnelle des acquisitions : le cas des habiletés motrices et de l'intervention en EPS. M. Quidu, Les Sciences du sport en mouvement, 2, (pp. 403-424).Paris : L'Harmattan.

Legrain, P., Arripe-Longueville, F., Gernigon, C. (2003). The influence of trained peer tutoring on tutors' motivation and performance in a French boxing setting. Journal of sports science, 21, 539-550.

Legrain, P., \& Heuzé, J. P. (2005). Intégration d'une tutelle réciproque entre pairs dans un dispositif collectif d'apprentissage en gymnastique : Etude de variables médiatrices. Symposium sur les relations entre pairs dans le domaine des activités physiques et sportives. Congrès International de la Société Française de Psychologie du Sport. Reims, France.

Mascret, N. (2008). Créer les conditions de l'interaction entre élèves difficiles en éducation physique et sportive: un exemple de forme de pratique scolaire du badminton. Thèse de doctorat non publiée. Université d'Aix-Marseille, Marseille.

Mirza, N. M., \& Perret-Clermont, A. N. (2008). Dynamiques interactives, apprentissages et médiations: analyses de constructions de sens autour d'un outil pour argumenter. In Processus interactionnels et situations éducatives (pp. 233-255). De Boeck Supérieur.

Mugny, G., \& Carugati, F. (1985). L'intelligence au pluriel: les représentations sociales de l'intelligence et de son développement. Cousset : Delval.

Perret-Clermont, A. N., \& Nicolet, M. (1988). Interagir et connaître. Enjeux et régulations sociales dans le développement cognitif. Cousset : Delval.

Piaget, J. (1976). La formation du symbole chez l'enfant. [Play, dreams, and imitation]. Neuchantel, Switzerland: Delachaux et Niestle.(Original work published 1946). 


\section{eJRIEPS spécial 2 (juillet 2019)}

Piaget, J., \& Inhelder, B. (1951). La genèse de l'idée de hasard chez l'enfant. Paris: Presses Universitaires de France. Englische über-setzt:(1975) The origin of the idea of chance in children. New York: Norton.

Radel, R., Sarrazin, P., Legrain, P., \& Wild, T. C. (2010). Social contagion of motivation between teacher and student: Analyzing underlying processes. Journal of Educational Psychology, 102(3), 570-580.

Rivière V. (2012). Spécificités et diversité des interactions didactiques. Lyon : Riveneuve éditions.

Rivière, C., \& Lafont, L. (2014). Favoriser l'inclusion d'élèves porteurs de troubles cognitifs par le tutorat en EPS. Carrefours de l'éducation, (1), 175-190.

Santini, J., \& Sensevy, G. (2010). Les interactions didactiques dans la dialectique jeux d'apprentissage-jeux épistémiques. Une étude de cas à l'école primaire. Colloque international Spécificités et diversité des interactions didactiques : disciplines, finalités, contextes. Université de Lyon-ICAR-CNRS-INRP, 24-26 juin.

Slavin, R. E. (1983). When does cooperative learning increase student achievement?. Psychological bulletin, 94(3), 429.

Sorsana, C. (1999). Stratégies socio-cognitives dans la résolution de la tour de Hanoï. In M. Gilly, J.-P. Roux, \& A. Trognon (Éds.), Apprendre dans l'interaction. Analyse des médiations sémiotiques (pp. 143-161). Aix-en-Provence et Nancy: Publications de l'Université de Provence et P.U.N.

Trognon, A. (1999). Éléments d'analyse interlocutoire. In M. Gilly, J.-P. Roux, \& A. Trognon (Eds.), Apprendre dans l'interaction. Analyse des médiations sémiotiques (pp. 69-94). Nancy et Aix-en-Provence : P.U.N. et Publications de l'Université de Provence.

Vergnaud, G. (1996). Au fond de l'apprentissage, la conceptualisation. Actes de l'école d'été de didactique des mathématiques, 174-185.

Vergnaud G. (1998). Les conditions de mise en œuvre de la démarche compétences. Communication aux Journées internationales de la formation. Centre national du patronat français (CNPF). Deauville, 7-8 octobre.

Vergnaud, G. (2002). Le schème est-il mort? Recherches en didactiques APS, EPS, STAPS, Impulsion 3, 11-26.

Vygotsky, L. (1985/1934). Pensée et langage. Paris : La Dispute (traduction par F. Sève). 